\title{
Le chant choral des jeunes filles à Sparte
}

Cadences poétiques, rythmes rituels, arts musicaux et identité sexuée Choral Songs of Young Women en Ancien Sparta: Poetic Forms, Ritual Rythms, Musical Arts and Gendered Identities.

\section{Claude Calame}

\section{(2) OpenEdition}

\section{Journals}

\section{Édition électronique}

URL : https://journals.openedition.org/clo/1935

DOI : 10.4000/clo.1935

ISSN : 2266-1816

\section{Éditeur}

INALCO

Édition imprimée

Date de publication : 1 janvier 2013

ISBN : 978-2-85831-217-7

ISSN : 0396-891X

Référence électronique

Claude Calame, "Le chant choral des jeunes filles à Sparte », Cahiers de littérature orale [En ligne], 73-74 | 2013, mis en ligne le 11 mai 2015, consulté le 01 juillet 2021. URL : http:// journals.openedition.org/clo/1935; DOI : https://doi.org/10.4000/clo.1935

Ce document a été généré automatiquement le 1 juillet 2021.

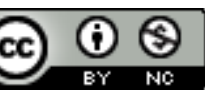

Cahiers de littérature orale est mis à disposition selon les termes de la Licence Creative Commons Attribution - Pas d'Utilisation Commerciale 4.0 International. 


\title{
Le chant choral des jeunes filles à Sparte
}

\author{
Cadences poétiques, rythmes rituels, arts musicaux et identité sexuée \\ Choral Songs of Young Women en Ancien Sparta: Poetic Forms, Ritual Rythms, \\ Musical Arts and Gendered Identities.
}

\section{Claude Calame}

Il y a une vengeance des dieux.

Heureux celui qui, avisé,

passe ses jours sans larmes.

Quant à moi, je chante (egòn d'aeído)

la lumière d'Agidô ;

je la vois qui s'élève comme le soleil ;

Agidô le conjure de paraître pour nous.

Mais l'illustre chorège ne me laisse

lui adresser ni louange ni blâme.

En effet elle me paraît elle-même se distinguer

autant que si, au milieu d'un troupeau de cavales,

on plaçait un destrier vigoureux,

victorieux aux jeux, au galop retentissant,

un cheval digne des rêves ailés.

1 Pour nous, en traduction, un texte poétique relevant de ce que nous dénommons «littérature grecque »; pour les Grecs anciens, l'une des strophes d'un poème rituel chanté par un groupe de jeunes filles sur un rythme musical et chorégraphique. Composé par un poète d'origine lydienne du nom d'Alcman pour être exécuté rythmiquement et rituellement - dans les circonstances cultuelles que l'on verra - par un chœur de jeunes filles appartenant aux familles aristocratiques de la Sparte du $\mathrm{VII}^{\mathrm{e}}$ siècle avant notre ère, ce poème exige non seulement, dans les différents rythmes qu'il implique, un effort de traduction transculturelle, conduisant de la trace textuelle à la forme discursive; mais ce chant requiert aussi une approche inspirée par l'ethnopoétique. Cette approche se double d'une sensibilité, animée par les sciences du 
langage, à la dimension énonciative du poème chanté et à sa pragmatique : l'une et l'autre sont marquées du point de vue rythmique ${ }^{1}$.

\section{Du rythme graphique aux rythmes vocaux}

2 Lorsque les Grecs anciens ont adapté l'alphabet phénicien à leur propre usage, ils ont introduit les voyelles dans un système essentiellement consonantique. Indépendamment du problème posé par le passage de la vocalisation d'une langue sémitique à une langue indo-européenne où les voyelles semblent un peu plus stables, une telle introduction a sans doute pour raison l'exigence de transcrire par ce moyen graphique les chants oraux de la poésie homérique. La diction homérique est en effet entraînée par un rythme vocal et métrique qui est fondé sur l'alternance des voyelles brèves et des voyelles longues. Ce n'est dès lors pas un hasard si les Grecs ont d'emblée pensé cette adaptation vocalique du système consonantique phénicien en termes de rythme. Dans une perspective historique et généalogique, Hérodote fait remonter cette adaptation au temps héroïque de l'émigration à Thèbes du héros Cadmos, accompagné de Phéniciens. À cette occasion, les Hellènes auraient reformulé les lettres " cadméennes », importées de Phénicie, du point de vue de la " prononciation » (phonée) et du point de vue de la «forme » (rhuthmós) ou plus littéralement, quant à la voix et quant au rythme ${ }^{2}$. De fait, quand en Grèce classique on lit un énoncé, on entend toujours la voix qui le prononce, avec sa cadence, son intonation et sa mélodie. C'est le cas jusque dans l'iconographie où les lettres sont toujours saisies dans la séquence phonique d'un énoncé conçu comme un flux (vocal); ceci conformément au sens étymologique de rhuthmós qui dérive de rheîn, "couler ». C'est ainsi que dans les Lois, Platon définit rhuthmós comme la cadence ordonnée (táxis) des mouvements du corps en relation avec la mélodie de la voix ; le flux harmonique de la voix est soutenu par l'alternance et l'accord des aiguës et des graves (Lois, 665a).

3 Dans le domaine musical donc, la cadence de la voix, quand elle assume une forme poétique, renvoie à un rythme chorégraphique, par la mesure et la mélodie de l'émission vocale. Or, destiné à une performance musicale dans des circonstances publiques et rituelles, le poème grec se présente souvent, du point de vue énonciatif, comme un acte de chant : les choreutes décrivent l'action musicale dans laquelle ils ou elles sont engagées. À la fois vocale, musicale et corporelle, marquée par un rythme métrique renvoyant à une chorégraphie, la pratique poétique grecque est donc justiciable de l'approche ethnopoétique indiquée. De la récitation des poèmes homériques à la représentation musicale et ritualisée de la tragédie, en passant par les différentes formes du mélos et du chant cultuel, la performance poétique hellène implique une "rythmicisation » rituelle et collective. À la fois organique et mental, ce rythme du poème chanté et dansé construit et entretient auprès des membres de la communauté, par la pratique musicale et rituelle, une identité culturelle et sociale ; une identité à la fois collectivement assumée et individuellement incarnée ; une identité qui relève d'un processus d' "anthropopoiésis ", c'est-à-dire de construction culturelle de l'humain ${ }^{3}$. Cette identité en consistance culturelle et sociale est traversée par des rythmes qui s'expriment dans la pratique rituelle et poétique - comme on le verra avec l'exemple emprunté à la Sparte préclassique - à plusieurs niveaux anthropopoiétiques. Aux rythmes qui traversent la performance chantée et dansée en ses différents niveaux de manifestation (cadence vocale, rythme de la mélodie musicale, rythme métrique 
correspondant à une chorégraphie et à une gestuelle, rythme strophique dans l'organisation du contenu) s'ajoute le rythme du calendrier annuel des célébrations cultuelles dont ces performances musicales rythmées sont un composant essentiel. Dans le cas des groupes choraux de jeunes filles spartiates, ces performances poétiques correspondent aux rituels marquant les moments initiatiques qui, avec leur cadence propre, conduisent de l'enfance à la maturité de l'adulte ${ }^{4}$ : rythmes métriques et chorégraphiques d'ordre musical, rythmes poétiques récurrents, rythme calendaire cyclique, mais pour un processus anthropopoiétique dynamique, impliquant quant à lui le changement de rythme.

Image $n^{\circ} 1$

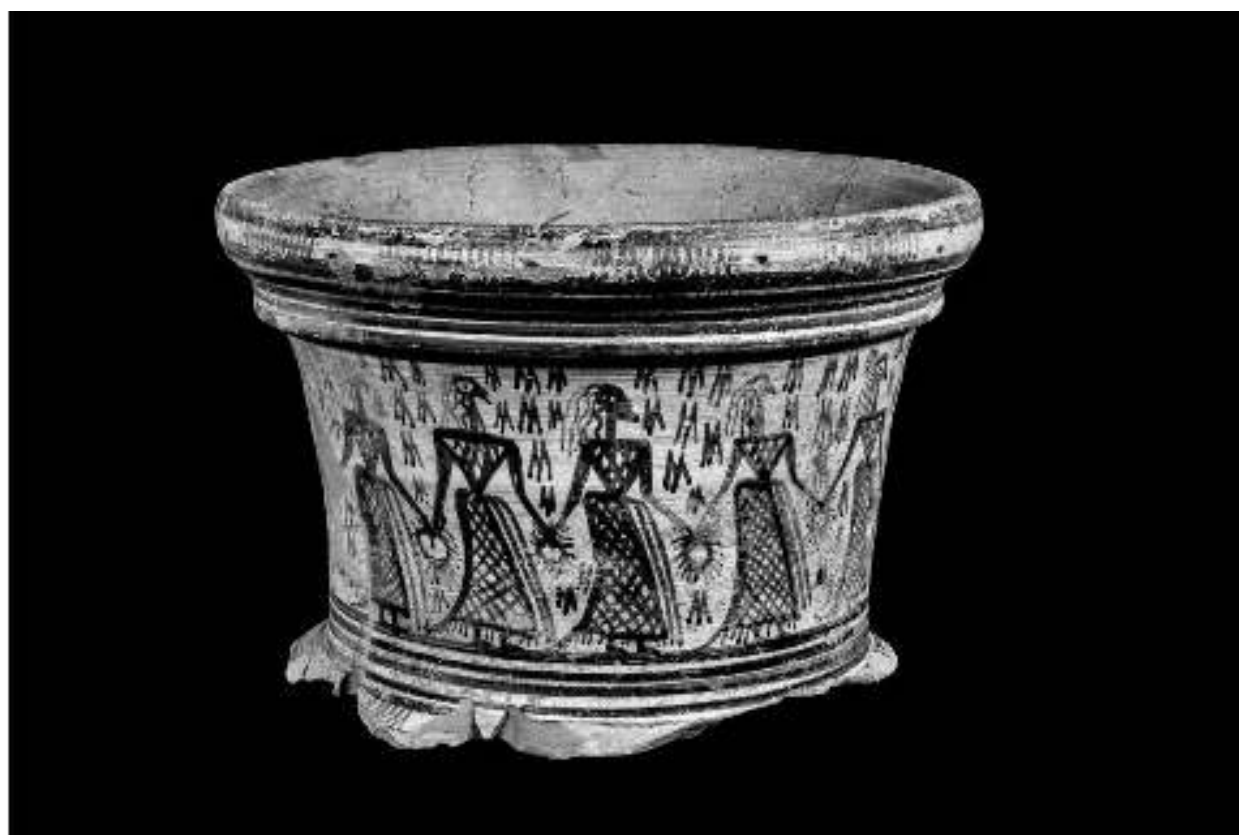

Chœur féminin (fın du vIII siècle av. J.-C.), col d'amphore funéraire, Érétrie (mE 6857). École suisse d'archéologie en Grèce.

\section{Rythmes anthropopoiétiques et rites d'initiation tribale}

4 En effet, en particulier dans la Lacédémone de l'époque préclassique, aussi bien les jeunes gens que les jeunes filles étaient soumis à un cursus initiatique dans lequel pratiques musicales et pratiques gymniques jouaient le rôle central que leur assignait l'éducation grecque traditionnelle. Pour les groupes choraux que formaient adolescentes et adolescents dans un probable système de classes d'âge, le parcours initiatique était ritualisé et scandé par leurs interventions musicales aux célébrations cultuelles rythmant le calendrier politique et religieux de la cité, dans un cycle annuel. De cette manière, par la récurrence calendaire et par le rythme cyclique des manifestations cultuelles destinées aux différentes divinités de la cité, les transitions impliquées par le développement biologique de l'adolescent ou de l'adolescente, en parallèle avec la construction de son identité sociale, religieuse et culturelle, étaient intégrées au rythme de la reproduction récurrente du corps social, avec son identité culturelle. 
5 Or dans les sociétés traditionnelles, parmi les rituels rythmés qui ont une fonction anthropopoiétique forte, les rites de passage réservés aux adolescentes et aux adolescents occupent une place de choix : ce sont eux qui assurent la construction identitaire et l'accès des jeunes au statut de membre adulte de la communauté sociale et culturelle. De ce point de vue, en particulier pour la poésie chantée appartenant à une culture historique dont le contexte ethnographique en général nous échappe (comme c'est le cas pour la Grèce antique), la comparaison anthropologique s'avère particulièrement fertile. Rappelons très brièvement que l'habitude salutaire a été prise, en anthropologie culturelle et sociale, d'inclure ces rites donnant accès au statut de l'adulte par la construction et la différenciation des identités, des rôles et des représentations de sexe, dans le sous-ensemble des « rites d'initiation tribale ». Cette spécification est d'autant plus importante que, pour la période de l'adolescence, il s'agit de distinguer le temps de la puberté biologique de la temporalité de la puberté sociale, l'un ne coïncidant que partiellement avec l'autre. Ainsi, pour les filles, plusieurs sociétés «traditionnelles» font le partage entre les rites de puberté, rites individuels liés à l'apparition des premières menstrues, et les rites d'initiation tribale à proprement parler, destinés de manière collective à une classe d'âge.

6 Le rite de passage et plus particulièrement le rite d'initiation tribale, sinon le rite de puberté sont donc à considérer et à utiliser comme des concepts opératoires et comparatifs $^{5}$. Ces concepts instrumentaux doivent permettre de saisir l'impact "anthropopoiétique» des performances musicales rythmées confiées aux groupes choraux d'adolescents ou d'adolescentes dans les cités de la Grèce "archaïque ». Chantées et dansées, elles contribuent à la construction d'une identité à la fois collective et individuelle par les différents rythmes qui les scandent, entre récurrences, tensions et ruptures. On parvient à les saisir en particulier dans l'un des chants rituels et choraux dont on a cité une strophe en guise de prélude. Par l'exercice collectif et rythmé des arts des Muses, les adolescentes du groupe choral qui chantait ces vers étaient conduites à la maturité sexuelle, sociale, culturelle et religieuse de la femme adulte, épouse du citoyen-soldat. Exécuté rituellement dans la Sparte de la fin du $\mathrm{VII}^{\mathrm{e}}$ siècle avant notre ère, ce chant est traversé par un entrelacs de rythmes qui concourent à sa fonction anthropopoiétique.

\section{Contexte institutionnel et culturel : une éducation initiatique}

7 Destiné à être chanté par des jeunes filles, le parthénée ${ }^{6}$ transmis de manière fragmentaire par le " Papyrus Mariette » (déposé au Louvre) a donc été composé par un poète sans doute originaire de Lydie, mais actif dans la Sparte de la fin du VII siècle et du début $\mathrm{du} \mathrm{VI}^{\mathrm{e}}$ avant notre ère. Or la Sparte de l'époque préclassique n'est pas la Lacédémone policée et militaire déjà idéalisée par Platon. Le pouvoir politique y est assumé par deux rois et contrôlé par cinq éphores; ces magistrats sont assistés du conseil des anciens qu'est la gerousía et ils sont choisis puis élus par l'apellá, l'assemblée du "peuple» réunissant les citoyens-soldats. Ceux-ci sont les membres d'une communauté civique qui est loin d'être homogène ; s'ils sont intégrés à la formation militaire de la phalange qui est marquée par la solidarité et l'égalité illustrées dans un célèbre poème élégiaque de Tyrtée, ils appartiennent à des familles de statut différent, parmi lesquelles les deux familles royales. Ces citoyens et soldats sont répartis dans les 
trois tribus «doriennes", à fonction apparemment militaire, qui sont également mentionnées par le poète plus ou moins contemporain d'Alcman qu'est Tyrtée : les Pamphyloi, les Hylleis et les Dymanes?

Les fils des citoyens, enfants, puis adolescents, étaient intégrés dans un système de classes d'âge, répartis en "troupeaux » (agélai) et en sous-groupes (hilai). Désigné dès l'Antiquité en tant que "conduite éducative " (agogé), ce système de formation à caractère initiatique a été comparé dès le début du $\mathrm{xx}^{\mathrm{e}}$ siècle aux Altersklassen connues par les communautés tribales et "primitives ». À la puberté, les adolescents étaient invités à choisir, parmi les néoi que sont les jeunes citoyens, un amant pour s'engager dans une relation pédérastique à fonction aussi bien éducative qu'initiatique : il ne s'agit pas de l'homosexualité selon la notion et l'étiquette moderne sous laquelle on place volontiers les relations grecques entre éromène et éraste, mais d'une homophilie qui en désigne le caractère fiduciaire d'un côté, mais transitoire de l'autre ${ }^{8}:$ donc une pédérotique à caractère rituel qui s'inscrit dans un cursus éducatif qu'on a comparé aux Jünglingsweihen des unzivilisierte Völker avant de lire ces pratiques selon le schéma ternaire du rite de passage forgé par Arnold Van Gennep'. L'ensemble institutionnel de l'agogé a dès lors été interprété en termes anthropologiques de classes d'âge et de rituels d'initiation tribale qui confèrent une scansion initiatique à la formation des adolescents spartiates $^{10}$. C'est notamment par la performance rituelle de formes musicales chantées et dansées que les adolescents soumis à ce cursus initiatique étaient associés à la célébration publique des dieux tutélaires de la cité comme Apollon d'Amyclées, Artémis Orthia ou Athéna "à la demeure de bronze ", dans des festivals à caractère politique tels les Carnéia, les Hyacinthies ou les Gymnopédies, une fête du " jeu nu » à caractère assurément initiatique ${ }^{11}$.

Il faut dire que la petite cité de Sparte connaît pendant toute la période préclassique un développement culturel et musical tout à fait remarquable. En témoignent non seulement une production céramique iconographique qui connaît une diffusion méditerranéenne, un artisanat raffiné d'objets en bronze, de nombreuses victoires remportées aux jeux panhelléniques d'Olympie, mais surtout un développement musical exceptionnel. Évoquant Sparte, le poète Pindare, dans des vers hélas fragmentaires, célèbre non seulement les assemblées des anciens et la valeur guerrière des jeunes citoyens, mais aussi les chœurs, la Muse et Aglaia, l'une des trois Grâces dont le nom dit l'éclat de la beauté12. Cela donc, à très grands traits, pour planter le décor ethnographique d'une cité quelque peu exotique, considérée déjà par Platon dans les Lois comme digne d'une approche anthropologique!

10 Mais qu'en est-il donc des adolescentes, filles de citoyens et futures épouses de Spartiates appartenant aux meilleures familles? Qu'en est-il des jeunes filles indirectement évoquées par Pindare et mises en scène un peu plus tard par Aristophane ou par Euripide? Quelle place leur est-elle réservée, du point de vue d'une éducation musicale à caractère initiatique et du point de vue de l'identité de sexe, dans une culture que l'on peut définir comme une culture politique de la performance poétique et rituelle, avec le réseau de rythmes pluriels, anthropopoiétiques qu'elle implique? 


\section{Le parthénée du « Papyrus Mariette » : pédagogie musicale}

11 Le poème spartiate transmis de manière fragmentaire par le « Papyrus Mariette » était composé de huit (ou neuf ?) strophes présentant un schéma métrique récurrent. Avec le retour circulaire qu'indique et qu'implique l'étymologie même du terme (de stréphein, "tourner ») ces longues strophes s'organisent en triades, c'est-à-dire en séquences de strophe-antistrophe-épode: soit deux strophes plus courtes, qui se répondent rythmiquement, et qui sont complétées par une partie conclusive dansée sur un rythme un peu différent. Ces unités et sous-unités rythmiques sont composées de séquences trochaïques et dactyliques, dans l'alternance d'une longue et d'une brève pour les premières, et d'une longue et de deux brèves pour les secondes. L'identification métrique du poème transforme d'emblée ce texte en une forme discursive orale, portée par une voix et un pas rythmés. Traduite en termes techniques de métrique, cette structure strophique, voire triadique, renvoie donc à une exécution chorale chantée et dansée, fort probablement sur l'accompagnement musical de la lyre $^{13}$.

12 Après une probable invocation aux Muses, le je poétique évoquait, sous la forme de la prétérition, une version spécifique du combat des Dioscures, les héros tutélaires de la cité, contre les fils d'Hippocoon; ces jeunes héros sont donnés par un commentateur ancien comme les rivaux en amour des cavaliers jumeaux que sont les Dioscures. Fils de Tyndare, le roi fondateur de Sparte, par leur filiation humaine, les Dioscures étaient les modèles héroïques du néos, le jeune citoyen-soldat en passe de se marier. Dans des vers également fragmentaires, la leçon est tirée du récit héroïque, comme c'est le cas dans tout poème mélique (selon la désignation indigène des poèmes que nous dénommons abusivement « lyriques ») :

(16)

(Qu'aucun) parmi les hommes

n'aspire au ciel ;

(qu'aucun) ne tente d'épouser Aphrodite.

13 Puis est évoqué un second épisode "mythique», sans doute en relation avec l'acte d'húbris des Hippocoontides mentionnés dans le premier "mythe", correspondant peut-être à la lutte des Tyndarides contre les fils d'Aphareus pour obtenir la main des Leucippides, c'est-à-dire les « blanches cavales ». Ce récit provoque un second et double énoncé gnomique. Au début de la quatrième strophe cette sentence, énoncée au présent, avec sa on-validité du point de vue énonciatif, assure le passage, attendu dans la poésie mélique grecque, du niveau du "récit» au niveau du «discours », pour reprendre les catégories opératoires proposées par le linguiste Émile Benveniste. Des énoncés du "mythe » à la troisième personne, à l'aoriste (avec son sens de temporalité à la fois passée et illimitée) et dans un lieu différent on passe aux énoncés marqués par l'« appareil formel de l'énonciation »: les formes en je/nous, dans l'instant et l'espace présents renvoient à l'hic et nunc de la performance même du poème. Les formes verbales autoréférentielles et performatives ( $\mathrm{au}$ sens propre du terme) qui transforment le poème en un acte de chant et un acte de culte jouent un rôle essentiel dans ce passage du « récit » au « discours » et dans la référence à la performance même du poème $\mathrm{e}^{14}$. 
14 C'est ainsi que le je poétique, qui correspond aussi bien au poète qui a composé ces vers qu'aux jeunes filles qui le chantent, poursuit son chant en énonçant la strophe citée en introduction. Les choreutes tirent la leçon du double récit mythique et paradigmatique qu'elles viennent de chanter avant de faire l'éloge, par un véritable acte de chant (egòn d'aeído, " quant à moi, je chante »; vers 39), de la lumineuse Agidô, puis de la jeune et belle femme qui assume la conduite du chœur. Dans le passage du je au nous, les choreutes prolongent leur louange poétique des deux jeunes femmes: l'une, Hagésichora, est leur chorège comme l'indique son nom propre alors que l'autre, Agidô, appartient peut-être par son nom à la famille royale des Agiades. Les deux jeunes femmes sont engagées dans un rituel que les comparaisons employées désignent comme une course :

(50)

Mais ne vois-tu pas?

D'un côté le coursier vénète ;

de l'autre la chevelure de ma cousine Hagésichora

fleurit comme de l'or pur.

Son visage d'argent,

pourquoi te le décrire dans sa lumière ?

C'est Hagésichora.

Et Agidô, seconde quant à la beauté,

court avec elle comme un cheval colaxéen

auprès d'une cavale ibène.

En effet, comme deux colombes,

elles combattent pour nous

qui portons à Orthria un voile,

s'élevant dans la nuit d'ambroisie comme l'astre Sirius.

15 De la description de la beauté de la chorège et de l'élan de la course dans laquelle la jeune Hagésichora est engagée avec Agidô, l'attention se porte dans la sixième strophe sur les jeunes filles qui forment le groupe choral. De la structure annulaire d'ordre sémantique qui clôt sur elle-même la cinquième strophe par une allusion à la vue et à la lumière d'Hagésichora, on passe à un nouveau mouvement de prétérition : changement de rythme non pas métrique, mais rhétorique. Assumant la position énonciative du je/ nous mélique, de manière autoréférentielle, les jeunes filles du chœur chantent :

(64)

Or, pour leur répondre,

la pourpre dont nous disposons ne suffit pas

ni un bracelet finement ciselé

dans l'or massif,

ni une mitre de Lydie,

ornement des jeunes filles au regard de violette,

ni les cheveux de Nannô,

mais pas davantage Aréta semblable aux dieux,

ni Thylakis, ni Cléésithéra,

et tu n'iras pas chez Ainésimbrota pour lui dire :

«Puisse Astaphis être mienne,

puisse Philylla me regarder,

et Damaréta, et la charmante Ianthemis ».

Mais c'est Hagésichora qui me poursuit.

En contraste avec l'imperfection et l'état en quelque sorte encore inachevé de chacune des choreutes, le je poétique reprend la louange des qualités d'Hagésichora dans la strophe suivante. Cette reprise établit une nouvelle structure annulaire d'ordre 
sémantique entre le début de cette septième strophe (vers 78) et le début de la cinquième strophe où Hagésichora est désignée deux fois par son nom propre (vers 52 et 56). L'éloge poétique induit, on va le voir, une évocation de la déesse de l'aube, Aôtis. C'est à cette divinité que le poème est sans doute destiné, dans sa performance même. Ces vers fournissent en somme la "clé " initiatique d'un chant rituel que son énonciation chorale et féminine en nous permet d'attribuer au genre mélique du parthénée :

(78)

En effet Hagésichora aux belles chevilles n'est pas ici, mais elle se trouve (auprès) d'Agidô :

elle approuve notre célébration.

Mais, (divinités,) recevez leurs (prières),

Car des dieux dépendent l'accomplissement

et le terme de toute chose.

Chorège, pourrais-je dire -

Mais quant à moi, adolescente,

je ne suis qu'une chouette qui s'égosille en vain,

perchée sur la poutre.

Pourtant je désire intensément plaire à Aôtis.

Elle est en effet le médecin de nos peines.

Mais c'est après leur séjour auprès d'Hagésichora

que les jeunes filles atteignent la paix aimable.

Après cette strophe qui rythmiquement est également organisée en un anneau d'ordre sémantique focalisé sur Hagésichora (vers 78 et 90), la louange poétique de la chorège se poursuit et s'achève dans la huitième et dernière strophe, malheureusement incomplète. Le rôle pédagogique attribué à la chorège se précise, incarné dans les qualités mélodieuses d'une voix qui évoque à nouveau la beauté érotique de la jeune femme :

(92)

En effet de même qu'au cheval de volée...

de même sur un navire aussi,

c'est au capitaine qu'il faut obéir.

Quant à elle, elle (n') a (pas) une voix plus mélodieuse

que les Sirènes,

car ce sont des déesses.

Au lieu de... dix enfants...

elle chante... comme le cygne sur les flots du Xanthos.

C'est elle qui, par sa chevelure d'or, inspiratrice du désir...

18 Ainsi les jeunes filles qui chantent le poème (dont le texte est ici à nouveau fragmentaire), en assumant son énonciation par un nous féminin, sont sensibles à la beauté physique de la chorège et de sa compagne, à la rapidité de leur pas de course et à la voix mélodieuse de celle qui, comme son nom propre l'indique, conduit le chœur. Dans l'usage d'expressions et de métaphores qui sont celles de la poésie mélique érotique rendue familière par les compositions de Sappho, d'Ibycos et d'Anacréon, le poème est traversé dans sa dynamique rythmée par trois lignes sémantiques qui en assurent à la fois la cohérence dans l'ordre du sens et la pragmatique référentielle. Dans ces trois isotopies (voix mélodieuse, agilité à la course, beauté physique), on retrouve non seulement l'expression poétique de la relation que les Grecs aimaient à établir entre la beauté harmonieuse d'une voix (volontiers féminine) et les effets de charme, sinon d'envoûtement érotique que provoque une telle vocalité. Mais ces trois lignes thématiques correspondent aussi aux trois piliers de l'éducation grecque traditionnelle 
tels qu'on les saisit par exemple dans les comédies d'Aristophane: la formation poétique et musicale, les exercices gymniques et les relations homoérotiques; masculines ou féminines, ces dernières sont marquées par l'asymétrie et par le constant décalage amoureux des rapports pédagogiques entre adolescents et adultes. Ce sont là aussi les trois composantes de l'éducation lacédémonienne destinée, à l'époque préclassique de la culture du chant, aux jeunes filles des meilleures familles ${ }^{15}$.

19 Mais du point de vue d'un gender entendu non seulement comme ensemble des relations et des identités sociales et symboliques de sexe, mais aussi comme ensemble des représentations que l'on s'en fait dans une culture particulière, un double paradoxe s'impose d'emblée. D'une part, le désir érotique et l'admiration amoureuse suscités par les deux jeunes femmes dont les choreutes font l'éloge sont chantés par des adolescentes dans des vers composés par un poète adulte et masculin. D'autre part, si ces vers expriment l'un des sentiments qui pour nous comptent parmi les plus intimes, ils sont chantés collectivement sur un rythme métrique et strophique dansé. Sur le plan énonciatif, ils sont assumés par l'ensemble des choreutes, dans le réseau des différents rythmes de la performance poétique. Or les commentateurs anciens des poèmes d'Alcman, édités à l'époque alexandrine, font du poète spartiate d'origine lydienne un " maître des filles et des éphèbes pour les chœurs ancestraux »; de plus, ils mettent en relation ce "maitre de chœurs", ainsi désigné dans sa "fonction-auteur ", avec des jeunes gens et des jeunes filles qui se révèlent appartenir aux deux familles royales de la Sparte contemporaine ${ }^{16}$. Ces quelques informations extérieures confirment la dimension éducative du poème dans sa performance musicale et rituelle elle-même, autant par l'exercice cadencé de la parole chantée que par l'entraînement rythmique du corps.

\section{La formation musicale et homoérotique à l'identité de sexe}

20 Les procédures d'énonciation du poème d'Alcman, dans leur dimension performative, ne sont pas les seules à être orientées par l'identité symbolique de sexe qui est celle de la jeune Spartiate appartenant aux familles aristocratiques de la cité. Cette identité est complémentaire à celle que l'éducation musicale, gymnique et érotique rythmée, dans un cursus initiatique spécifique, confère au futur citoyen : épouse d'un citoyen et mère d'un côté, acteur politique et soldat de l'autre. Cette orientation marquée du point de vue du gender traverse toute la matière sémantique du poème, et ceci à plusieurs niveaux d'expression :

- du point de vue du récit héroïque que nous appréhendons comme «mythe ", autant le combat des Dioscures, fils de Tyndare, contre les Hippocoontides que la probable allusion à l'affrontement des deux jeunes héros aux Apharétides ont pour enjeu la conquête de jeunes filles nubiles ;

- la leçon qui est tirée du premier épisode «mythique » donné en exemple fait explicitement allusion au mariage, envisagé quant au statut de l'épouse ; l'húbris serait ici de prétendre épouser Aphrodite. De plus, dans ce passage très fragmentaire, la déesse semble être associée aux Charites, les Grâces « dont le regard inspire l'amour »; cette qualification est en quelque sorte explicitée par un vers d'Hésiode qui renvoie à l'idée de la force érotique émanant de la personne aimée pour être véhiculée par son regard ${ }^{17}$; 
- comme on aura encore à le préciser, la divinité à laquelle s'adressent indirectement les jeunes choreutes est une déesse attachée, par sa beauté séductrice, au moment du passage de l'adolescente à l'âge adulte, précisément signifié par l'intervention du désir érotique comme force de fécondation et de création ;

- et comme cela a été signalé, si les mots chantés ont été composés par un poète mâle et adulte, l'instance d'énonciation correspondant au je poétique est adossée à une posture auctoriale collective, marquée par la féminité et l'adolescence.

21 En rapport avec les identités de sexe et les relations sociales qui en découlent, il s'agit ici de construire les représentations de ces identités et relations ; il s'agit de les mettre en acte dans et par la pratique poétique et rituelle rythmée. Ainsi la poésie érotique grecque s'avère être, dans et par sa performance cadencée, un puissant moyen de construction anthropopoiétique des identités et des relations marquées par le sexe. Dans le cas particulier, par la performance chantée et dansée, dans l'accomplissement rituel impliquant le corps et la voix, les jeunes choreutes acquièrent une identité féminine à la fois individuelle et sociale. La participation anthropopoiétique au groupe choral conduit les jeunes filles spartiates à la maturation sexuelle (par l'exercice poétique du désir érotique), à la maturation affective et physique (en particulier par le rythme de la danse) et à la maturité civique (ne serait-ce que par l'évocation des mythes fondateurs de l'ordre civique spartiate, focalisés sur leurs enjeux matrimoniaux). C'est donc par l'intégration rythmique au groupe choral comparable à une sous-classe d'âge, par la participation à la formation chorale avec son caractère rituel, et par les performances musicales cadencées à l'occasion des grands festivals scandant la vie civique et religieuse de la communauté civique que les jeunes filles des familles aristocratiques de Sparte accèdent au statut d'épouse de citoyen et de mère des futurs soldats, avec le changement de rythme de vie que cela implique pour elles.

Or, dans des strophes qui sont elles-mêmes rythmées par un enchaînement marqué et cadencé notamment par les structures annulaires, les jeunes choreutes chantent poétiquement le désir érotique suscité par le visage, le mouvement et la voix non pas d'un jeune homme égal aux Dioscures, mais de la jeune chorège (tout en exprimant leur admiration pour son associée). La référence est ici à une relation homoérotique entre chacune des adolescentes participant au groupe choral et la jeune femme qui en assume la conduite: relation amoureuse que nous placerions sous l'étiquette de l'«homosexualité » (féminine), mais que j'ai dit préférer qualifier $d^{\prime}$ " homophilie $»^{18}$; relation érotique transitoire dans la mesure où elle conduit la jeune fille à la sexualité pleinement assumée dans le mariage qui conduit, avec l'aide de plusieurs divinités parmi lesquelles Aphrodite, à la maternité.

Ainsi en va-t-il à vrai dire, du côté masculin, des relations entre éraste et éromène ; bien avant le Banquet de Platon, le second recueil des distiques élégiaques attribués à Théognis nous offre de nombreux témoignages de ce rapport amoureux à fonction éducative entre un adulte amant et un adolescent aimé. Marquée par l'intervention d'Éros, cette relation est asymétrique, puisqu'elle s'établit entre un homme confirmé dans son statut de citoyen et un adolescent qui vient d'accéder à la puberté ; elle est transitoire dans la mesure où elle est attachée et limitée au moment de passage et de changement du statut biologique et social que signifie l'adolescence ; elle est formative puisqu'elle est conçue comme une éducation esthétique par la pratique poétique au désir érotique qui est ensuite satisfait dans une relation hétérosexuelle. De plus, elle renforce la relation pédagogique essentielle du jeune éromène avec un éraste qui, dans 
la philía comme estime réciproque, constitue le modèle du jeune homme pour les vertus masculines. Enfin, notamment dans le cadre du symposion, cette relation amoureuse est marquée par le décalage constitutif d'un désir érotique que seul l'adulte peut pleinement satisfaire. Avec son caractère collectif et rituel, la relation d'homophilie masculine assure, notamment par la performance poétique rythmée, le passage initiatique vers l'éros de l'adulte.

Dans cette mesure, le rapport homophile masculin peut être rapproché des pratiques d'éros féminin qui s'inscrivent en filigrane dans le parthénée choral d'Alcman : relation asymétrique entre les choreutes qui ne disposent pas encore de tous les moyens de la séduction et Hagésichora qui exerce sur ces jeunes filles un charme érotique certain autant par l'éclat de son visage que par sa voix mélodieuse ${ }^{19}$; relation transitoire dans la mesure où les choreutes disent leur aspiration à atteindre à leur tour la beauté marquant la maturité sexuelle (par exemple vers 74-76); relation formative puisque la position de la chorège par rapport aux choreutes est explicitement comparée à celle du cheval de volée ou à celle du capitaine de navire (vers $92-94)^{20}$; relation collective et rituelle dès lors qu'elle s'exprime, de manière autoréférentielle, chorale et symbolique, dans la performance musicale et rituelle rythmée des mots poétiques chantés ; relation homophile en décalage, puisque les choreutes disent, avec leur voix de chouette (vers 86-87), ne pas encore disposer de tous les atours susceptibles d'exercer la séduction qui est celle de leur chorège. Femme dans tout l'éclat de la beauté à peine parvenue à sa maturité, Hagésichora est seule susceptible de permettre aux jeunes filles de son groupe choral d'atteindre la «paix aimable » (vers 91). La métaphore désigne ici l'achèvement des "peines » de l'éducation chorale pour parvenir, collectivement et individuellement, à la pleine maturité de la condition féminine, du point de vue physique, social et symbolique ${ }^{21}$.

Ainsi, si le parthénée du Papyrus Mariette ne peut être attribué à une phase précise d'un rite d'initiation tribale destiné aux jeunes filles de Sparte, sa performance rituelle chorale, avec ses différents rythmes poétiques et musicaux, s'inscrit assurément dans un cursus anthropopoiétique à caractère et à fonction initiatiques; elle inscrit le rythme de développement "gynéco-poiétique » des jeunes filles lacédémoniennes des familles aristocratiques dans le rythme cyclique des célébrations cultuelles de la cité.

Mais qu'en est-il enfin de ces circonstances cultuelles du chant choral composé par Alcman?

\section{Hélène entre adolescence et statut de la femme adulte}

Deux figures divines sont évoquées par les jeunes filles du chœur d'Alcman: Orthria comme déesse du Matin et Aôtis comme déesse de l'Aube. En relation avec le moment de la cérémonie qui semble se situer au lever du soleil comme l'indique la comparaison initiale d'Agidô avec sa lumière (vers 40-43), ces deux noms sont sans doute à considérer comme des épiclèses renvoyant toutes deux à Hélène.

Hélène était en effet honorée à Sparte dans deux cultes distincts, comme héroïne et comme divinité. D'une part, Pausanias nous signale auprès du Dromos, où se déroulaient les courses des jeunes hommes (néoi) sous l'égide des Dioscures, un lieu ombreux dit Platanistas. Bordée de platanes et entourée d'un canal qui en faisait une île, cette esplanade accueillait les combats rituels des éphèbes que le législateur Lycurgue passait pour avoir institués. Auprès du Platanistas s'élevaient plusieurs 
sanctuaires héroïques édifiés pour quelques-uns des fils d'Hippocoon, et pour honorer Héraclès ainsi qu'Hélène. C'est d'ailleurs auprès de ce sanctuaire d'Hélène, sans doute vénérée en tant qu'héroïne encore jeune fille, que se trouvait la tombe du poète Alcman $^{22}$. D'autre part, à Thérapné, dans le sanctuaire qui s'élevait au-dessus de l'Eurotas sur les restes d'un édifice mycénien, Hélène bénéficiait avec son époux Ménélas d'un culte en tant que divinité; elle y était vénérée comme femme adulte susceptible de conférer aux jeunes filles, sous le signe d'Aphrodite, la beauté marquant l'accès à la nubilité, au mariage et à la maternitén ${ }^{23}$. Le double culte dont Hélène bénéficiait à Sparte offre une oscillation entre les qualités de la jeune fille prête à affronter le mariage et le statut de l'épouse pourvue des séductions de la beauté féminine achevée ; cette polarité n'est pas sans rappeler la tension que l'on a évoquée entre les choreutes encore adolescentes du parthénée d'Alcman et leur chorège parvenant ou parvenue à la maturité sexuelle et sociale de l'adulte. Sans qu'il soit possible de déterminer à quelle occasion précise était destiné le chant parthénée d'Alcman, la présence d'Hélène dans le poème ne fait que confirmer le passage rituel auquel contribue sa performance chorale, elle-même ritualisée et rythmée.

L'exécution chantée et dansée du poème d'Alcman par les jeunes choreutes spartiates contribue donc au passage rituel des jeunes filles qui le chantent à l'identité de sexe biologique, sociale et symbolique de la femme adulte. Elle s'inscrit non pas dans un rite de passage ou un rite d'initiation tribale à proprement parler, mais dans un processus initiatique et rituel rythmé, d'ordre anthropopoiétique. En effet sa performance ne correspond pas à l'état de mort symbolique qui caractérise la phase liminaire du rite d'initiation tribale et qu'on a voulu retrouver dans de nombreux mythes grecs mettant en scène adolescentes ou adolescents ${ }^{24}$. Mais ces pratiques rituelles et musicales rythmées contribuent à construire des identités (de sexe) à la fois individuelles et collectives dans un processus d'éducation initiatique politique, religieux et culturel cadencé ; ce processus polyrythmique se développe à partir d'un inachèvement et d'une incomplétude que les choreutes ne manquent pas d'exprimer ${ }^{25}$.

En faisant de la performance musicale un acte rituel, en insérant celle-ci dans une célébration cultuelle à la cadence cyclique, en assurant le passage de l'état transitoire de l'adolescente à celui plus permanent de l'adulte, les rythmes métriques et rhétoriques du parthénée d'Alcman contribuent à l'insertion anthropopoiétique des jeunes filles qui chantent le poème dans leur nouveau rythme de vie biologique, culturel et social de femmes adultes, en particulier du point de vue de la puissance productrice et créatrice que représente Éros : épouses, puis mères.

\section{BIBLIOGRAPHIE}

AFFERGAN, Francis, BORUTTI, Silvana, CALAME, Claude, FABIETTI, Ugo, KILANI, Mondher et REMOTTI, Francesco, 2003, Figures de l'humain : les représentations de l'anthropologie, Paris, Éditions de l'EHESS. ARRIGONI, Giampiera, 1983, Donne e sport nel mondo greco. Religione e società, in Giampiera Arrigoni (ed.), le Donne in Grecia, Roma-Bari, Laterza, p. 55-201. 
BENVENISTE, Émile, 1974, Problèmes de linguistique générale II, Paris, Gallimard.

BETTINI, Maurizio et BRILLANTE, Carlo, 2002, il Mito di Elena : Immagini e racconti dalla Grecia a oggi, Torino, Einaudi.

BOEHRINGER, Sandra, 2007, l'Homosexualité féminine dans l'Antiquité grecque et romaine, Paris, Les Belles Lettres.

BoWIE, Ewen, 2011, Alcman's first Partheneion and the Song the Sirens Sang, in Lucia Athanassaki and Ewen Bowie (eds), Archaic and Classical Choral Song. Performance, Politics and Dissemination, Berlin, de Gruyter, pp. 33-65.

BRELICH, Angelo, 1969, Paides e parthenoi, Roma, Ateneo.

BRELICH, Angelo, 2008, le Iniziazioni, Roma, Editori Riuniti.

CALAME, Claude, 1977, les Chœurs de jeunes filles en Grèce archaïque, vol. II, Alcman, Roma, Edizioni dell'Ateneo.

CALAME, Claude, 1983, Alcman : introduction, texte critique, témoignages, traduction et commentaire, Roma, Edizioni dell'Ateneo.

CALAME, Claude, 1993, Rythme, voix et mémoire de l'écriture en Grèce classique, in Roberto Pretagostini (ed.), Tradizione e innovazione nella cultura greca da Omero all'età ellenistica. Scritti in onore di Bruno Gentili, I, Roma, Gruppo editoriale internazionale, p. 785-799. Repris dans 2008, Sentiers transversaux : entre poétiques grecques et poétiques contemporaines, Grenoble, Jérôme Millon, p. 205-216.

CALAME, Claude, 2001, Choruses of Young Women in Ancient Greece (transl. by D. Collins and J. Orion), Lanham \& Oxford, Rowman \& Littlefield (2nd ed.) [éd. or., 1977, les Chœurs de jeunes filles en Grèce archaïque, vol. I, Morphologie, fonction religieuse et sociale, Roma, Edizioni dell'Ateneo].

CALAME, Claude, 2002, Interprétation et traduction des cultures : les catégories de la pensée et du discours anthropologiques, l'Homme, 163, p. 51-78.

CALAME, Claude, 2003, Modes rituels de la fabrication de l'homme : l'initiation tribale, in Francis Affergan et al., 2003, Figures de l'humain : les représentations de l'anthropologie, Paris, Éditions de l'EHESS, p. 129-173.

CALAME, Claude, 2004, Identités d'auteur à l'exemple de la Grèce classique : signatures, énonciations, citations, in Claude Calame, Roger Chartier (éds), Identités d'auteur dans l'Antiquité et la tradition européenne, Grenoble, Jérôme Million, p. 11-39.

CAlAmE, Claude, 2009, l'Éros dans la Grèce antique, Paris, Belin ( $3^{\mathrm{e}}$ éd.).

CALAME, Claude, 2010a, Fiction référentielle et poétique rituelle : pour une pragmatique du mythe (Sappho 17 et Bacchylide 13), in Danièle Auger et Charles Delattre (éds), Mythe et Fiction, Paris, Presses de Paris Ouest, p. 117-135.

CALAME, Claude, 2010b, Les rites d'initiation tribale et le passage à l'âge adulte dans mythes et rituels grecs : une perspective d'anthropologie historique et critique, in Philippe Hameau (éd.), les Rites de passage : de la Grèce d'Homère à notre XXI siècle, Grenoble, Musée dauphinois, p. 121-132.

CALAME, Claude, 2012, Arts des Muses et poètes citoyens : la Sparte archaïque comme culture du chant, in Vincent Azoulay, Florence Gherchanoc, Sophie Lalanne (éds), le Banquet de Pauline Schmitt : genre, mours et politique dans l'Antiquité grecque et romaine, Paris, Publications de la Sorbonne, p. 399-426. 
CALAME, Claude, DUPONT, Florence, LORTAT-JACOB, Bernard et MANCA, Maria, 2010, la Voix actée : pour une nouvelle ethnopoétique, Paris, Kimé.

CARTLEDGE, Paul, 2001, Spartan Reflections, London, Duckworth.

DOWDEN, Ken, 1989, Death and the Maiden. Girls'Initiation Rites in Greek Mythology, London-New York, Routledge.

DUCAT, Jean, 2006, Spartan Education: Youth and Society in the Classical Period, (transl. by E. Stafford, P. J. Shaw and A. Powell), Swansea, The Classical Press of Wales.

DUPONT, Florence, 2010, Introduction, in Claude Calame, Florence Dupont, Bernard Lortat-Jacob, Maria Manca (éds), la Voix actée : pour une nouvelle ethnopoétique, Paris, Kimé, p. 7-20.

GRAF, Fritz, 2003, Initiation. A Concept with a Troubled History, in David B. Dodd \& Christopher

A. Faraone (eds.), Initiation in Ancient Greek Rituals and Narratives. New Critical Perspectives, LondonNew York, Routledge, pp. 3-24.

HODKINSON, Stephen, 1999, An Agonistic Culture? Athletic Competition in Archaic and Classical Spartan Society, in Stephen Hodkinson \& Anton Powell (eds), Sparta. New Perspectives, London, Swansea, Duckworth, The Classical Press of Wales, pp. 147-187.

Michon, Pascal, 2007, les Rythmes du politique. Démocratie et capitalisme mondialisé, Paris, Les Prairies ordinaires.

POWELl, Anton, HODKINSON, Stephen (eds), 2002, Sparta. Beyond the Mirage, Swansea, London, Duckworth, The Classical Press of Wales.

RICHER, Nicolas, 2012, la Religion des Spartiates : croyances et cultes dans l'Antiquité, Paris, Les Belles Lettres.

VAN GENNEP, Arnold, 1909, les Rites de passage, Paris, E. Nourry.

\section{NOTES}

1. Pour l'ethnopoétique, on verra la définition nouvelle donnée par DUPONT (2010). Une version différente de ce texte a été présentée au colloque vigmA de l'université de Strasbourg en novembre 2011.

2. HÉRODOTE 5, 58; voir à ce propos mon étude détaillée de 1993/2008 avec de nombreuses références bibliographiques à propos de l'adaptation au grec de l'alphabet phénicien.

3. Sur cette notion d'ordre opératoire, voir les différentes recherches publiées dans AFFERGAN et al. (2003).

4. Comme je l'ai proposé dans l'enquête doctorale de 1977, mise à jour dans la traduction anglaise de 2001. Lectrices et lecteurs voudront bien excuser les nombreuses références bibliographiques aux travaux que j'ai déjà consacrés au poème d'Alcman allégué ici.

5. Pour le caractère instrumental de ces deux concepts, voir les deux études critiques de CALAME $(2003,2010 \mathrm{~b})$, notamment quant à leur fonction opératoire et comparative pour les pratiques de l'Antiquité grecque; mise au point historique utile par exemple dans l'essai de BRELICH (2008, sur la base d'un cours donné en 1959-1960). 
6. Pour l'histoire de cette forme poétique et mélique chorale, cf. CALAME $(1977,149-176)$.

7. Cf. TYRTÉE, fr. 9,15-26 et fr. 10,16 GENTILI-PRATO; les très nombreux problèmes soulevés par notre compréhension historique et sociale des institutions politiques de la Sparte préclassique et classique sont évoqués en particulier dans les études réunies par CARTLEDGE (2001); voir aussi celles réunies par POWELL et HODKINSON (2002).

8. Les deux témoignages fondamentaux quant à l'agogé spartiate sont ceux de XÉNOPHON, République des Lacédémoniens $(2,2-13)$ et de PLUTARQUE, Vie de Lycurgue $(16,7-17,8)$; voir à ce propos, en dernier lieu, DUCAT $(2006,1-67)$. Pour les relations d'homophilie et leur caractère initiatique, je renvoie aux propositions que j'ai formulées en 2009 (133-145), en revisitant l'idée de la Dorische Knabenliebe et de ses différentes interprétations.

9. VAn GenNeP $(1909,14$ et 27), pour le schéma tripartite articulant les rites de séparation, les rites de marge et les rites d'agrégation; ou, dans les termes mêmes proposés par le grand folkloriste français: rites préliminaires, rites liminaires, rites postliminaires.

10. Voir en particulier BRELICH $(1969,113-207)$ et DUCAT $(2006,69-118)$, pour un réexamen critique des informations à disposition; parcours historique de l'interprétation du cursus éducatif spartiate en termes d'initiation tribale chez CALAME (2003, 149-157), avec les références bibliographiques de rigueur; voir encore à ce propos la contribution critique de GRAF (2003).

11. Documentation sur ces trois festivals politico-religieux chez CALAmE (2001, 141-206) et maintenant chez RICHER (2012,323-456 pour les Carnéia, 343-382, pour les Hyacinthies, 383-422 pour les Gymnopédies) ; voir aussi DUCAT (2006, 249-280).

12. PINDARE, fr. 199 MAEHLER; voir aussi les témoignages choraux d'ARISTOPHANE, Lysistrata (1296-1312) et d'EURIPIDE, Hélène (1465-1477). Les nombreux témoignages sur la song culture qu'offre la Sparte préclassique font l'objet du commentaire de CALAME (2012) ; pour la culture athlétique de Lacédémone et la participation des Spartiates aux jeux panhelléniques, voir par exemple l'étude de HODKINSON (1999).

13. alcman, fr. 1 page-davies $=3$ calame. Pour toutes les questions de commentaire technique de ce poème, l'auto-référence est, une fois pour toutes, à mon travail philologique de 1983, en particulier (219-220) pour la structure métrique du poème et (311-312), sur son attribution du poème à Alcman ; pour le commentaire sémantique, voir CALAME $(1977,43-133)$; bibliographie complémentaire et interprétation un peu différente dans l'étude de BOWIE (2011).

14. Voir les concepts opératoires d'analyse des discours utilisés une fois encore pour l'étude pragmatique de deux poèmes méliques dans le bref essai de CALAME (2010a); c'est en particulier le cas de "l'appareil formel de l'énonciation» emprunté à BENVENISTE (1974, 79-88).

15. Pour l'érotisme de la voix poétique, cf. CALAME $(2009,56-60)$; les métaphores de poésie érotique dans le parthénée du «Papyrus Mariette " sont décrites par CALAME (1977, 86-97) ; sur le rôle de la course et de l'athlétisme dans l'éducation spartiate destinée aux jeunes filles, voir ARRIGONI $(1983,65-95)$; sur la fonction pédagogique des relations homoérotiques grecques, voir les références données dans CALAME (2009, 119-175). 
16. $\mathrm{Cf}$. Alcman, fr. $\mathrm{S} 5$ Page-Davies $=$ test. 13 Calame et fr. 10 (b) Page-Davies $=82$ Calame, avec le commentaire correspondant dans CALAME (1983, XVI-XXI et 454-461). Emprunté à Michel Foucault, le concept de « fonction-auteur » est particulièrement opératoire pour signifier la position énonciative et sociale du poète, cf. CALAME (2004).

17. Cf. HÉSIODE, Théogonie (910-911: «De leurs paupières coulait l'éros qui rompt les membres ; beau est le regard qu'elles lancent sous leurs sourcils »); autres références chez CALAME $(2009,31-38)$.

18. Pour une critique positive du concept moderne d'homosexualité en particulier dans le domaine féminin et pour l'Antiquité gréco-romaine, voir les remarques introductives à l'enquête conduite par BOEHRINGER (2007, 17-34), qui se fait l'avocate d'une "historicisation» de la sexualité ; à la nécessité de la perspective historique j'ajouterai celle de l'approche anthropologique : «traduction transculturelle » dans le temps et dans l'espace (CAlAme, 2002, 60-78); pour l'«homophilie», cf. CALAME (2009, 122-145).

19. Si le mouvement de prétérition qui marque la sixième strophe (vers 64-77) est positif, comme le remarque BOWIE (2011, 40-42), cela n'empêche nullement les choreutes de se donner comme inférieures à la chorège, en particulier en ce qui concerne la voix : elles se comparent à une chouette qui s'égosille en vain (vers 86-87), cf. CALAME (1983, 324-343).

20. Voir le commentaire de CALAmE (1983, 345-346).

21. Sur le sens de cette " paix ", cf. calame $(1977,118-119 ; 1983,344-345)$.

22. PAUSANIAS, 3, 14, 6 et 8 ainsi que 15,1-3; références bibliographiques chez CALAME (2001, 193-196) ; voir de plus BETTINI et BRILLANTE (2002, 43-55).

23. Voir à nouveau PAUSANIAS, 3,19,9-10, ainsi qu'ALCMAN lui-même, fr. 7 PAGEDAVIES = 19 CALAME; les autres témoignages sur ce culte d'Hélène à Therapné sont commentés dans CALAME (2001, 193, 196-198 et 200-202).

24. Pour les jeunes filles, voir par exemple l'étude de DOWDEN (1989).

25. Michon $(2007,37-88)$, a bien souligné le rôle joué par les rythmes dans les processus d'individuation.

\section{RÉSUMÉS}

La culture du chant de la Grèce ancienne et particulièrement celle de la Sparte préclassique sont riches de poèmes méliques correspondant à des chants choraux exécutés rituellement à l'occasion des différentes étapes d'un cursus d'éducation érotique, religieuse et sociale à caractère initiatique. Aux rythmes qui traversent la performance chantée et dansée en ses différents niveaux de manifestation (cadence vocale, rythme de la mélodie musicale, rythme métrique correspondant à une chorégraphie et à une gestuelle) s'ajoute le rythme du calendrier annuel dans lequel s'insèrent les rituels marquant les moments initiatiques qui, avec leur cadence propre, conduisent de l'enfance à la maturité de l'adulte. C'est le propos que peut 
illustrer un poème "parthénée » composé par le poète spartiate Alcman et destiné à un groupe choral de jeunes filles.

The song culture of Ancient Greece, particularly in preclassical Sparta, offers many melic poems corresponding to choral songs ritually performed on the occasion of the different stages of an educational cursus, erotic, religious and social with an initiatory character. To the different rhythms of the sung and danced performance (vocal rhythm, rhythm of the musical melody, metrical rhythm corresponding to a choreography and a set of gestures) has to be added the rhythm of the annual civic calendar in which are inserted the cultic festivals with the rituals attached to the different stages leading young men and young girls from adolescence to mature adulthood. This rythmical sung and ritual cursus can be highlighted in the reading of a "partheneion" song composed by the poet Alcman in Sparta and performed by a choral group of young women.

INDEX

Index géographique : Grèce

Keywords : Ehnopoetics, Female Songs (Partheneia), Ritual of Initiation, Sparta, Rhythm, Alcman Thèmes : ethnopoétique

Mots-clés : chants féminins (parthénées), rite d'initiation, Sparte, Alcman

\section{AUTEUR}

\section{CLAUDE CALAME}

École des hautes études en sciences sociales, Paris 